\title{
The misleading pulse
}

\section{Bhushan Sudhakar Wankhade, Sananta Kumar Dash, Sudha Kansal, Rajesh Chawla}

A 49-year-old woman, a treated case of pulmonary tuberculosis, was admitted to our hospital with shortness of breath. She had a grade IV systolic murmur at the lower left sternal border, and her ECG showed P-pulmonale in lead II. Computed tomography of her chest revealed bilateral fibrosis and bronchiectatic changes. She was drowsy, hypoxic, and hypotensive. She was intubated for acute respiratory acidosis. A bedside two dimensional echocardiogram revealed severe pulmonary hypertension $(89 \mathrm{~mm} \mathrm{Hg})$ and severe tricuspid regurgitation (TR). The right internal jugular vein (IJV) and right femoral artery (most prominent pulsation in right femoral neurovascular bundle) were cannulated. We suspected misplacement of the arterial line due to similar mean pressures $(24 \mathrm{~mm}$ $\mathrm{Hg}$ ) and blood gas values of both catheters (Table). Misplacement of the line was diagnosed with ultrasonography (USG), which showed a pulsatile femoral vein with abnormal flow pattern (Figure 1) and a femoral artery with normal triphasic flow (Figure 2). Subsequently the lateral pulsatile structure (femoral artery) was cannulated under USG guidance, and this showed an arterial wave form and a BP $80 / 40 \mathrm{~mm}$ of $\mathrm{Hg}$. Arterial placement was confirmed by blood gas analysis.

Table Blood gas analysis

\begin{tabular}{|l|c|c|c|c|c|}
\hline Parameters & $\mathbf{p H}$ & $\mathbf{P a C O}_{\mathbf{2}}$ & $\mathbf{P a O}_{\mathbf{2}}$ & $\mathbf{H C O}_{\mathbf{3}}$ & $\mathbf{S a O}_{\mathbf{2}}$ \\
$\mathbf{( \mathbf { m m }} \mathbf{H g})$ & $\mathbf{( m m} \mathbf{H g})$ & $(\mathbf{m e q} / \mathbf{L})$ & $\mathbf{( \% )}$ \\
\hline IJV sample & 7.12 & 61 & 29 & 22 & 35 \\
\hline $\begin{array}{l}\text { Misplaced } \\
\text { line sample }\end{array}$ & 7.21 & 58.9 & 25 & 22.8 & 33.8 \\
\hline $\begin{array}{l}\text { Arterial line } \\
\text { sample }\end{array}$ & 7.23 & 46.1 & 61 & 19.1 & 87 \\
\hline
\end{tabular}

Corresponding author: Sananta Kumar Dash Contact Information: drsananta@yahoo.co.in DOI: 10.12746/swrccc2013.0103.035

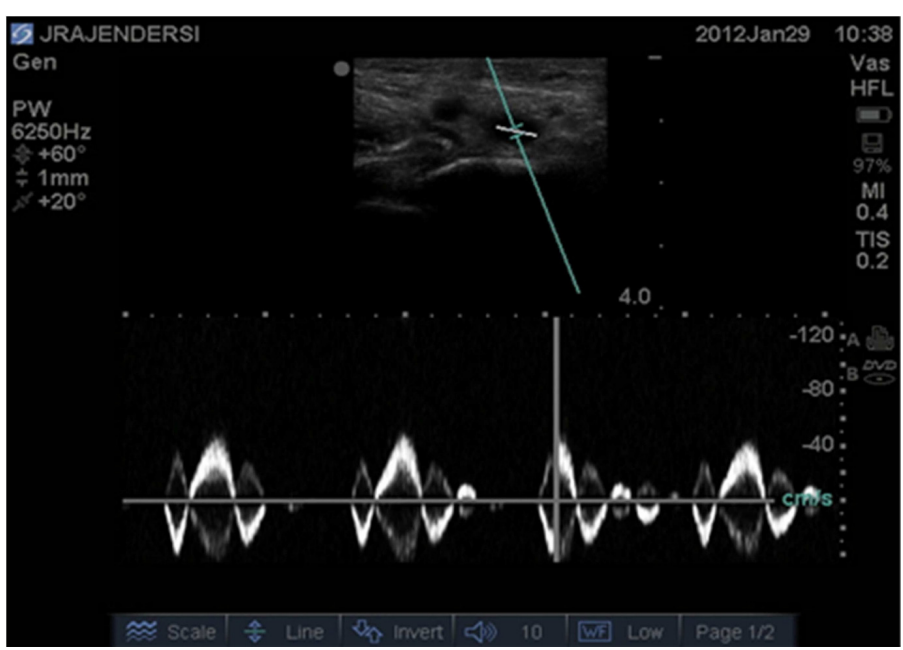

Figure 1. Doppler ultrasonography of femoral vein showing unusual phasicity.

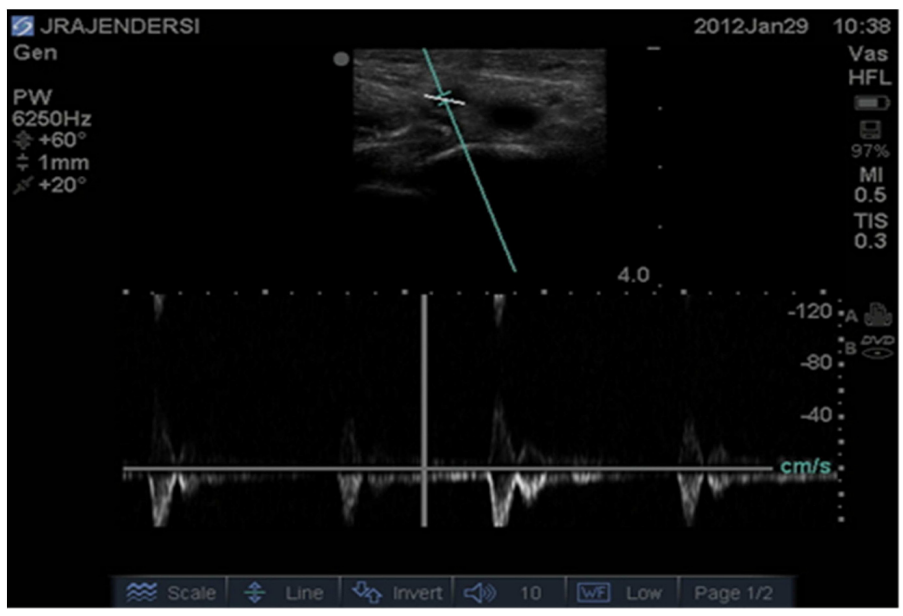

Figure 2. Doppler ultrasonography of femoral artery showing characteristic triphasic Doppler signal.

TR can occur in long standing mixed (obstructive and restrictive) pulmonary disease ${ }^{1}$. Our patient had post pulmonary tuberculosis related mixed airway disease. TR can cause arterialization of the femoral vein and a dilemma in the distinction between artery and vein ${ }^{2}$. The conventional anatomic relationship between the femoral vessels is not always depend- 
able ${ }^{3}$. This may cause inadvertent placement of the femoral arterial line into the femoral vein and subsequent inappropriate use of a vasopressor due to erroneous BP measurement ${ }^{4}$.

Normal flow characteristics of the femoral vein have been described ${ }^{5}$.These are summarised by the following mnemonic, "CAPSULE" : 1) Colour fill: no colour filling defects, 2) Augmentability: increase flow with distal augmentation manoeuvre, 3) Phasicity: phasic variation with respiration, i.e., a fall in venous flow velocity with inspiration and rise with expiration, 4) Spontaneity: flow observed at quiescence, 5) Unidirectional flow in cephaloid direction due to venous valves, 6) Loss of pulsatility, 7) Even flow contour. The normal femoral arterial flow pattern shows a characteristic triphasic Doppler signal with a fast upstroke to peak systole, a reversal of blood flow during early diastole, and a forward flow component during late diastole (Figure 2).

In our patient, there were marked venous pulsations secondary to TR, and arterial pulsations were not palpable due to profound shock. This led to the erroneous placement of a femoral arterial cannula into the femoral vein. It was diagnosed after comparing arterial and mixed venous oxygen saturation and later confirmed by USG. It showed an absence of augmentability and presence of phasicity, flow turbulence, pulsatility, and uneven flow contour of the femoral vein. Anatomical variation and pathophysiological changes in vasculature can lead to misplacement of invasive lines and misinterpretation of data. This can easily be diagnosed by ultrasonography, and we recommend routine use of ultrasonography for placement of all invasive lines. Physicians performing invasive cannulations and monitoring should be aware of normal ultrasonographic flow patterns to distinguish between artery and vein. We suggest a simple pneumonic (CAPSULE) to summarise the normal flow pattern in the femoral vein.

KEY WORDS- Tricuspid Regurgitation, Arterialization of vein.
Author Affiliation: Bhushan Sudhakar Wankhade and Sananta Kumar Dash are senior registrars and Fellows of national board of examination FNB, Department of Respiratory and Critical Care Medicine, Indraprastha Apollo Hospitals, New-delhi, India. Sudha Kansal and Rajesh Chawla are Senior Consultants, Department of Critical Care Medicine, Indraprastha Apollo Hospital, New Delhi, India.

Received: 02/08/2013

Accepted: 05/09/2013

Reviewers: Cihan Cevik, MD, Kenneth Nugent, MD.

Published electronically: 7/15/2013

Conflict of Interest Disclosures: None

\section{REFERENCES}

1. Ahmed AEH, Ibrahim AS, Elshafie SM. Pulmonary hypertension in patients with treated pulmonary tuberculosis: analysis of 14 consecutive cases. Clin Med Insights Circ Respir Pulm Med 2011; 5:1-5.

2. Appelberg, Gaylis H. Pulsating veins in the lower extremities caused by tricuspid incompetence. S Afr Med J 1973; 47:1261-2.

3. Sharma p, Salwan s. Duplication of femoral vein and its significant clinical implications. International J Anat Variations 2011; 4: 188-91.

4. Gilron I, Magder S. Monitoring complication due to a pulsatile femoral, vein from tricuspid regurgitation. Can J Anaesth $1995 ;$ 42:141-3.

5. Necas M. Duplex ultrasound in the assessment of lower extremity venous insufficiency. Australasian J Ultrasound Med 2010; 13: 37-45. 